\title{
A new optimal energy management strategy based on improved multi-objective antlion optimization algorithm: applications in smart home
}

\author{
Mehdi Ramezani ${ }^{1} \cdot$ Danial Bahmanyar $^{1} \cdot$ Navid Razmjooy $^{2}$ (D)
}

Received: 16 August 2020 / Accepted: 12 November 2020 / Published online: 25 November 2020

(c) Springer Nature Switzerland AG 2020

\begin{abstract}
In recent years, energy demand has grown significantly relative to its production. The power companies have also offered a variety of schemes such as energy consumption management to meet this growing consumer demand. Energy consumption management is a set of strategies used to optimize energy consumption which includes a set of interconnected activities between the utility and customers to transfer the load from peak hours to off-peak hours. This reduces the electricity bill. This paper presents an optimal schedule for the consumption of residential appliances based on improved multi-objective antlion optimization algorithm to minimize the electrical cost and the user comfort. To prevent peaks, the peak-to-average ratio is considered as a constraint for the energy cost function. Also, two different tariff signals have been used to measure energy costs. The real-time pricing and critical peak pricing are considered as energy tariffs. The simulations results are compared with other meta-heuristic algorithms, including multi-objective particle swarm optimization, the second version of the non-dominated sorting genetic algorithm, and the basic antlion optimizer algorithm to show the superiority of the proposed algorithm. Final results show that using the proposed scheme reaches electricity bills less than $80 \%$.
\end{abstract}

Keywords Energy demand · Energy optimization · Energy consumption · Improved antlion optimization · Multiobjective optimization algorithm

\begin{tabular}{llll} 
Abbreviations & & \multicolumn{2}{l}{ List of symbols } \\
IMOALO & Improved multi-objective antlion & $S$ & The set of shiftable appliances \\
MOALO & optimization & $t$ & Time slot \\
MOPSO & Multi-Objective Antlion Optimization & $T$ & Number of time slots \\
& optimization & $\mathrm{DL}$ & Energy consumed during a day \\
NSGAII & The second version of the non-domi- & $\mathrm{HL}(t)$ & Energy consumed at time slot $t$ \\
& nated sorting genetic algorithm & $S_{i}$ & On or off state of ith appliance \\
PAR & Peak-to-average ratio & $P_{i}$ & Power rate of $i$ th appliance \\
RTP & Real-time pricing & Price $(t)$ & Energy price at time slot $t$ \\
CPP & Critical peak pricing & Load $_{\text {peak }}$ & The most load consumed at a time at a \\
DSM & Demand-side management & Load $_{\text {avg }}$ & certain time slot \\
HEMS & Home energy management system & PAR $_{\text {unscheduled }}$ & PAR value for the unscheduled pattern \\
& & $\beta$ & Penalty factor
\end{tabular}

$\triangle$ Navid Razmjooy, navid.razmjooy@hotmail.com | ${ }^{1}$ Department of Electrical and Control Engineering, Tafresh University, 3951879611 Tafresh, Iran. ${ }^{2}$ Departamento de Engenharia de Telecomunicações, Universidade Federal Fluminence, 25086-132 Rio de Janeiro, Brazil. 


\begin{tabular}{|c|c|}
\hline$V$ & Violation \\
\hline & User's ideal time for the ith appliance \\
\hline
\end{tabular}

\section{Introduction}

Electricity is a very valuable source of energy, and the need for it is growing every day. Due to the limitations of traditional energy resources, two kinds of solutions have been considered for them: discovering alternative energy sources to generate more energy and making effective use of available resources. The second approach is more desirable and more economical [1]. Problems and challenges in increasing the capacity of electricity generation and increasing demand for electricity have led various countries around the world to seek an appropriate solution to optimize energy consumption [2]. The main challenges facing the traditional power grids today are the possibility of blackouts, lack of liquidity, the security of transmission lines, water crisis, and the environment. Since misusing or loss of energy and these grids cannot overcome these problems, using smart grids (SG) is a good idea for solving these kinds of problems [3]. SG includes a set of interconnected activities between the utility and consumers to rationalize electricity consumption so that the same efficiency in the field of electricity can be achieved with more efficiency and lower cost. Using SG, power consumption can be controlled through smart meters that create a two-way consumption between the consumer and the source [4]. Demand-side management (DSM) is one of the solutions to these problems, which has been introduced by electricity companies to control energy consumption by consumers [5-7]. The main goal of DSM is to create a balance between supply and demand for providing mainly two tasks: consumption reduction and efficiency improvement [8]. DSM includes productivity programs and demand response programs. In demand response programs, consumers are invited to participate in incentive schemes. These plans are based on changes in electricity prices. Therefore, consumers can change their consumption patterns accordingly. By doing so, their electricity bills will be significantly reduced $[9,10]$. In traditional grids, utility manually sheds the consumer loads during peak hours to secure equipment on transmission lines. However, load shifting on the smart grids is done during peak hours to prevent peak load and damage to equipment. This is profitable for the company and the consumers and also increases network reliability [11]. Responding to such challenges requires meta-heuristic optimization algorithms that are highly capable of solving multi-objective optimization problems to manage energy consumption.
There are several related works in the literature, for example, genetic algorithms have been used in [12] to transfer power consumption from peak hours to off-peak hours to reduce power consumption. Of course, its application in the smart grid requires a calculation that must be done by cloud computing. It was said that this issue will be analyzed in the future.

An improved differential evolution algorithm was proposed in [13] to reduce energy cost, increase user comfort, and reduce PAR. Using renewable resources was also suggested to increase efficiency. However, their proposed algorithm still needed to be improved due to computational formulas.

A smart energy management system was introduced in [14]. The peak loads and the cost of electricity in a residential area were minimized by using multi-objective mixed-integer linear programming (MOMILP). Constraints included daily energy needs and consumer preferences. This system brought possible benefits to the company and the consumer.

In [15], a home energy management controller was proposed based on genetic harmony algorithm to reduce PAR and electricity cost and increase user comfort. To evaluate the performance of the proposed system, a single home and multiple homes with critical peak pricing signal and real-time pricing signals were considered.

A multi-period artificial bee colony (MABC) was used to increase productivity and reduce operating costs [16]. They used a real-time approach to manage the load. Management of energy consumption patterns in the residential area was possible to optimize various home appliances. It should be noted that in the proposed system, user convenience was not considered.

The community-based participatory energy plan was discussed in [17]. The authors examined the cost of consumer electricity bills and the smart grid. The main goal was to reduce PAR and electricity costs. This was done by connecting the consumer and the smart grid. The proposed plan was evaluated using the community-to-community plan in MATLAB. However, there was a compromise between user convenience and security.

In [18], micro-grid was introduced as an important part of the distribution system. The authors suggest the use of stand-alone hybrid renewable energy systems that are environmentally safe and economically efficient as a suitable solution for areas where electricity is not sufficiently available. They have presented technical and economic research for a residential area using the mentioned system. Their design included batteries, diesel, wind, and photovoltaics.

Achieving possible solutions in a shorter period to manage the demand side was identified as a challenge in [19]. Energy cost and user comfort have been considered as 
two important goals for load management, and to achieve these goals, a hybrid non-dominated sorting genetic algorithm has been used. However, the authors ignore the peak-to-average ratio.

In [20], a dragonfly meta-heuristic optimization algorithm is used to optimize the consumption of shiftable appliances taking into account the RTP pricing signal. Reducing energy costs and PAR and increasing user comfort are the goals ahead. By achieving these goals, network stability is also achieved.

However, different types of classic and bio-inspired solvers have been applied for determining the optimal solution of the problem, each of them has its shortcomings from premature convergence to their speed run and trapping in the local minimum.

In [18], an improved version of a meta-heuristic optimization algorithm called the improved emperor penguin optimization algorithm (IEPO) is used to optimize the performance of a cooling, heating, and combined power system.

The authors propose a multi-objective smart home energy management system for intelligently controlling small-scale load demand in [19]. Their objective is to reduce energy costs and reduce peak demand. They also use cooperative game theory to find the optimal Pareto solution to the multi-objective problem.

Meta-heuristic optimization algorithms have a very high ability to solve multi-objective optimization problems. It has been seen that these algorithms have better solutions for managing energy consumption. However, each of these algorithms has its disadvantages. We present an improved version of the MOALO algorithm to cover these disadvantages as much as possible.

This paper presents a home energy management system (HEMS) for achieving an optimal schedule for the consumption of residential appliances. This is done based on DSM in the smart grid. The improved multi-objective antlion optimizer algorithm as a new and efficient bioinspired algorithm is proposed to reduce the cost functions. Then, the results are compared with three other algorithms including MOPSO, NSGAIl, and MOALO. RTP and CPP are two electricity pricing scheme which is used in this study. In this paper, two objective functions are considered: electricity cost function with PAR constraint to reduce energy consumption and prevent the formation of peak load to increase grid efficiency and waiting time function to increase user comfort.

\section{Problem statement}

The DSM increases the reliability and stability of the SG performance while reducing energy consumption costs. This is the most important issue in the SG that needs special attention. In a smart home, the energy management system controls energy consumption by scheduling residential appliances [21]. In [22], a scheme has been proposed that reduces the cost of electricity consumption and, on the other hand, tries to increase consumer comfort. However, there is a trade-off between cost and consumer comfort. The DSM requires two-way communication of information between the consumer and the utility. This information includes the pricing signals set by the utility and the amount of consumer demand. The HEMS also plans the device consumption pattern based on the same information and user setting. The authors present a model of the HEMS in [23]. They suggested meta-heuristic methods for efficient energy consumption optimization accordingly to the RTP and IRB pricing signals.

Reducing the cost of energy consumption is the main goal of this study. Achieving this goal is possible using the DSM. Due to electricity tariffs, the cost of electricity consumption increases during peak hours. Therefore, consumption management reduces electricity bills by transferring the peak load of consumption from peak hours to off-peak hours. This increases the waiting time and thus reduces the user comfort, but this is not the only challenge ahead. Transfer of consumption load to off-peak hours causes peak formation during these hours, so the proposed solution should be able to establish a trade-off between reducing costs and increasing user comfort, as well as prevent peak load formation by reducing PAR.

\section{Problem classification}

\subsection{Load categorize}

There are several appliances in a home that is different in terms of power rating, performance, and operational time. In the proposed model, the appliances are divided into three categories of shiftable, non-shiftable, and fixed. Figure 1 shows a simple design of the proposed system model. According to this figure, there is two-way communication between the consumer and the utility. The operational time of shiftable appliances can shift at any time during a day. These appliances have a special role for the efficiency of the proposed design because by changing the operational time of these appliances from peak hours to off-peak hours, the cost of electricity and PAR can be significantly reduced, but this puts the user's comfort at risk. The issue of user comfort requires that these devices start operating at an ideal time in the user's view. Nonshiftable are those appliances whose operational time cannot be shifted. Fixed appliances are named because their operation length is unknown and also these devices start operation according to user demand. The details of 
Fig. 1 The suggested method arrangement

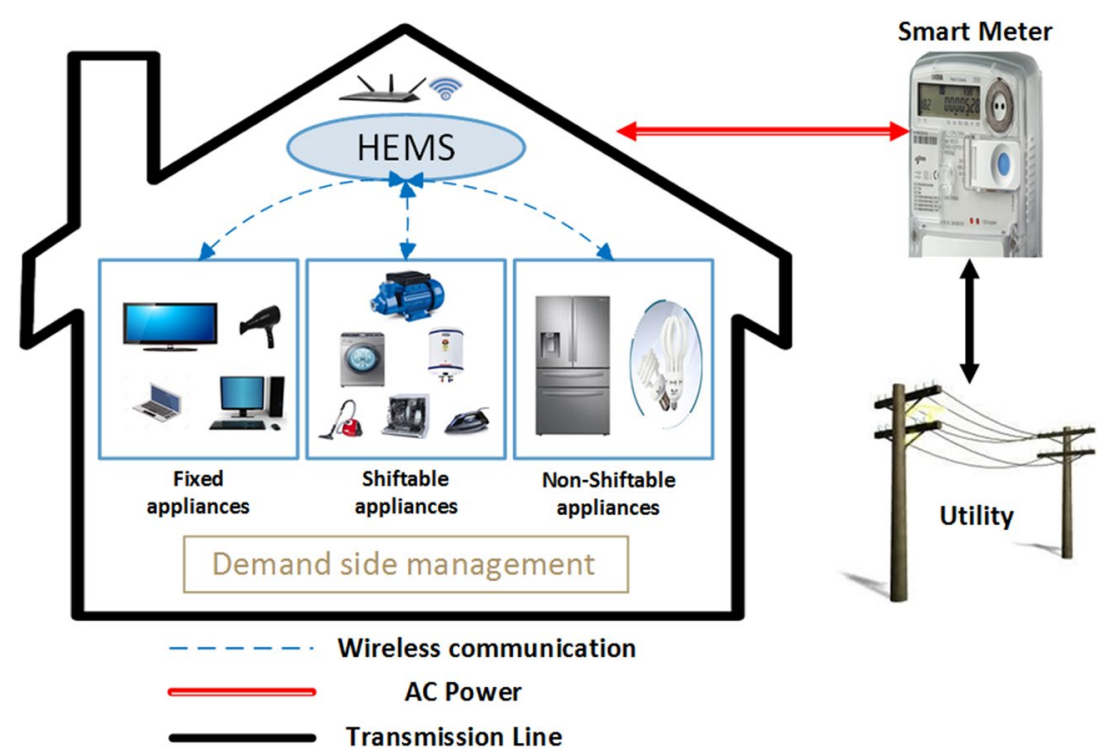

Table 1 Appliances classification

\begin{tabular}{lllll}
\hline Appliances & $\begin{array}{l}\text { Load catego- } \\
\text { rize }\end{array}$ & $\begin{array}{l}\text { Operational } \\
\text { time (hours) }\end{array}$ & $\begin{array}{l}\text { Power } \\
\text { rate } \\
(\mathrm{kW})\end{array}$ & $\begin{array}{l}\text { Ideal } \\
\text { starting } \\
\text { time }\end{array}$ \\
\hline Water heater & Shiftable & 10 & 2.6 & - \\
Water pump & & 10 & 2 & - \\
Vacuum & 2 & 1.2 & $09: 00$ \\
$\quad$ cleaner & & 3 & 2.5 & $22: 00$ \\
Dishwasher & & 1 & 1.2 & $06: 00$ \\
Steam iron & & 2 & 3 & $17: 00$ \\
Washing & & 1 & 3.4 & $19: 00$ \\
$\quad$ machine & & 22 & 0.3 & - \\
Dryer & & 7 & 0.4 & - \\
Refrigerator & Non-shiftable & 22 & 0.1 & - \\
Lights & & 2 & 0.3 & - \\
Laptop & Fixed & & 0.3 & - \\
PC & & 5 & 1.2 & - \\
TV & & 1 & 0.3 & - \\
Hairdryer & & & & \\
Blender & & 2 & & \\
\hline
\end{tabular}

this category, with the power rate and operating time of each appliance, are given in Table 1.

\subsection{Electricity tariff}

The electricity company uses various electricity tariffs to calculate the daily electricity costs of consumers. TOU, DAP, RTP, and CPP are examples of these tariffs. In this paper, the RTP and CPP tariff is used to calculate the cost of energy. In the RTP method, pricing is determined based on customer demand, so the price of electricity is constantly changing throughout the day. It may even be different every few minutes. Using this pricing method requires smart meters to establish effective two-way communication between the consumer and the company. The CPP tariff, like the TOU, has fixed prices at various periods, but depending on the critical events that occur in the system, the price can change for at least a period. This pricing scheme is not economically suitable for consumers, but it can be used to significantly reduce the burden in times of crisis [20]. To reduce the consumption in critical hours of the year when electricity consumption is very high and the reliability of the system is at risk, using CPP seems to be a good solution. Therefore, it can be said that the purpose of CPP is to increase the reliability and the stability of the power grid. Figure (2) shows the CPP and RTP signals.

\subsection{Cost functions}

In this study, a home with a set of appliances is considered. Each day is divided into twenty-four-time slots. Each interval represents 1 hour. The proposed model works based on the schedule of shiftable appliances. The main objectives of this study are to reduce the electricity costs by scheduling energy consumption during off-peak hours and to reduce PAR to increase grid reliability in a way that increases the user's comfort as much as possible.

Shiftable appliances:

$S \in\left[s_{1}, s_{2}, \ldots, s_{N}\right], N=7$

Time slots:

$t \in T=[1,2, \ldots, T], T=24$

where $S$ shows the shiftable appliances, $N$ is the number of shiftable appliances, and $\mathrm{t}$ is the number of the time slot. 
Fig. 2 Pricing signals [21]

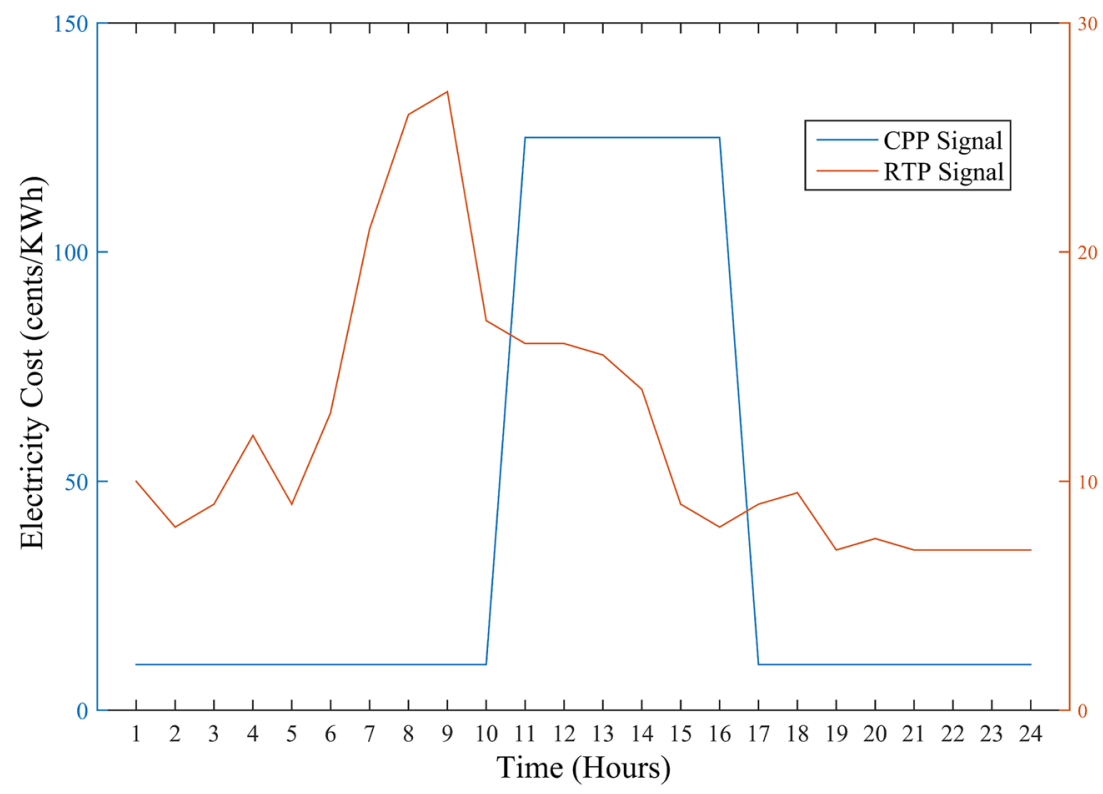

Electricity costs are calculated using RTP and CPP pricing tariffs and energy consumption by the appliances. The energy consumed during a day is given as:

$\mathrm{DL}=\sum_{t=1}^{T} \mathrm{HL}(t)$

where DL shows the total load demanded during 1 day, $\mathrm{HL}(t)$ represents the energy consumed at time slot $t$, and $T$ is the maximum of time slots.

Using the pricing signals, the daily energy cost is given as follows:

MinCost $=\sum_{t=1}^{T}\left(\sum_{i=1}^{N}\left(s_{i} \times P_{i}\right)_{t} \times \operatorname{Price}(t)\right)$

where $s_{i}$ indicates the on or off state of $i$ th appliance, $P_{i}$ represents the power rate of each appliance, and Price $(t)$ is the energy prices at time $t$.

The PAR is defined as the ratio of the maximum load consumed by a consumer over a specified time slot during a day and the average total load during the same day. As PAR increases, the reliability of the grid decreases, and its stability is compromised. However, reducing PAR not only increases the stability of the grid, but also reduces the cost of electricity to consumers, and therefore, both the consumer and the utility benefit from the implementation of such schemes. Mathematically, it is computed as follows:

$\operatorname{Load}_{\text {peak }}=\underset{t \in T}{\max (\mathrm{HL}(t))}$
$\operatorname{Load}_{\mathrm{avg}}=\frac{\sum_{t=1}^{T} \mathrm{HL}(t)}{T}$

where $\mathrm{HL}(t)$ represents the energy consumed at time slot $t$ and $T$ is the maximum of time slots.

According to Eqs. (5) and (6), PAR is given as:

PAR $=\frac{\operatorname{Load}_{\text {peak }}}{\text { Load }_{\text {avg }}}=\frac{\max (\underset{t \in}{\mathrm{HL}(t))} \times T}{\sum_{t=1}^{T} \mathrm{HL}(t)}$

where Load $_{\text {peak }}$ represents the most load consumed at a certain time slot, and Load $_{\text {avg }}$ represents the average load consumed during a day.

The unscheduled pattern reduces the cost of energy consumed by transferring the peak load consumed from peak hours to off-peak hours, but the peak load consumption may occur during off-peak hours. To prevent this from happening, the following constraint is considered:

constraint : PAR $<$ PAR unscheduled

where PAR $R_{\text {unscheduled }}$ is the PAR value for the unscheduled pattern.

We apply this constraint as a multiplicative penalty function to the electricity cost function. Therefore, the electric energy cost function changes as follows:

MinCost $=\left(\sum_{t=1}^{T}\left(\sum_{i=1}^{N}\left(s_{i} \times P_{i}\right)_{t} \times \operatorname{Price}(t)\right)\right) \times(1+\beta \times \tanh (v))$

where $\beta$ is the penalty factor, and here, $\beta=10$; also $v$ is the violation and the tanh (.) the function is used to bound the violation between $[0,1]$ The violation is defined as follows: 
$v=\max \left(\frac{\mathrm{PAR}}{\mathrm{PAR}_{\text {unscheduled }}}-1,0\right)$

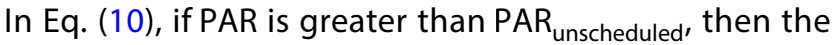
violation is positive. Otherwise, the violation is considered zero.

User comfort is defined based on consumer waiting time. Table 1 shows the user's ideal time to start the shiftable appliances. Reducing the cost of energy consumption and PAR increases the waiting time and endangers the user's comfort. Therefore, by reducing the waiting time, user comfort can be increased. The waiting time is calculated as follows:

WaitingTime $=\frac{\sum_{i=1}^{N} \mid \text { time }_{\text {ideal }}^{i}-\text { time }_{\text {start }}^{i} \mid}{N}$

where $N$ represents the number of shiftable appliances, here $N=7$, time ${ }_{i d e a l}^{i}$ is the user's ideal time for the ith appliance, and time ${ }_{\text {start }}^{i}$ is the starting time for the $i$ th appliance.

\section{Algorithms}

Optimization or programming in mathematics, economics, management, and engineering refers to selecting the best member from a set of achievable members. In the simplest form, an attempt is made to select the data from an achievable set and calculate the value of a function of its maximum and minimum value. Classical or analytical methods such as linear programming (LP) [22] seek to solve problems accurately. Therefore, they include differentiation to find the optimal answer. The main advantage of this type of optimization algorithms is to ensure the optimal answer, but they are difficult to use in problems of high complexity or large problems or with a discrete function. Although heuristic optimization algorithms do not guarantee the achievement of the most optimal and accurate answer and provide answers close to the exact answer, they can solve complex and difficult problems. The convergence speed of such algorithms is very high and they are applied in all fields of engineering.

The problem presented in this paper is a nonlinear and complex discrete problem, so it cannot be solved using classical methods. Therefore, we have proposed the improved multi-objective antlion optimization algorithm to achieve an optimal scheme for residential appliances in a way that reduces the cost of electricity, PAR, and waiting time.

\subsection{Basic ALO algorithm}

Before explaining the multi-objective version of the ALO algorithm, the base version of this algorithm must be provided. The ALO algorithm is based on the hunting mechanism of antlions and the principles inspired by their interaction with ants. In this algorithm, there are two populations: ants and the antlions. The main responsibility of the ants is to explore the search space using random walking. The antlions maintain the best position obtained by the ants and update their position as the ant's position improves. There is also an elite antlion in this search space that affects the movement of ants, regardless of its distance to other ants. If any of the antlions finds a better position than the elite, it will be replaced. After reaching the condition of stopping, the position of the elite antlion is selected as the final optimal response [23]. To model the interactions between ants and antlions, we first consider the ants to move in search space; then, the antlions are allowed to hunt. The random movement of ants is modeled according to the following equation:

$$
\begin{aligned}
X(t)= & {\left[0, \text { cumsum }\left(2 r\left(t_{1}\right)-1\right), \text { cumsum }\left(2 r\left(t_{2}\right)-1\right), \ldots,\right.} \\
& \text { cumsum } \left.\left(\operatorname{tr}\left(t_{n}\right)-1\right)\right]
\end{aligned}
$$

where cumsum is the cumulative sum, $n$ represents the maximum number of iteration, $t$ shows the step of random walk, and $r(t)$ is a stochastic function that is calculated as follows:

$r(t)=\left\{\begin{array}{l}1 \text { if rand }>0.5 \\ 0 \text { if rand } \leq 0.5\end{array}\right.$, rand $\in[0,1]$

Since any search space has its limitations, the range of variables in Eq. (13) cannot be used directly to update the position of ants. For the ants to walk randomly, their movement is normalized according to Eq. (14):

$X_{i}^{t}=\frac{\left(X_{i}^{t}-a_{i}\right) \times\left(d_{i}^{t}-c_{i}^{t}\right)}{\left(b_{i}-a_{i}\right)}+c_{i}^{t}$

where $a_{i}$ represents the minimum random walk of the $i$ th variable and $b_{i}$ is the maximum random walk, $d_{i}^{t}$ is the maximum of $t$ th variable at $t$ th iteration, and $c_{i}^{t}$ indicates 
the minimum of $i$ th variable at $t$ th iteration. Antlion pits are simulated as Eq. (15):

$\left\{\begin{array}{l}c_{i}^{t}=\text { Antlion }_{j}^{t}+c^{t} \\ d_{i}^{t}=\text { Antlion }_{j}^{t}+d^{t}\end{array}\right.$

In this equation, Antlion ${ }_{j}^{t}$ represents the $j$ th antlion at $t$ th iteration. A roulette wheel is used to model the ability of ants to hunt. When an ant is trapped in a pit, the antlion throws stones at the edges of the pit. Its mathematical model is as follows:

$\left\{\begin{array}{l}c^{t}=\frac{c^{t}}{l} \\ d^{t}=\frac{c^{t}}{l}\end{array}\right.$

In Eq. (16) / is a ratio and is defined as follows:

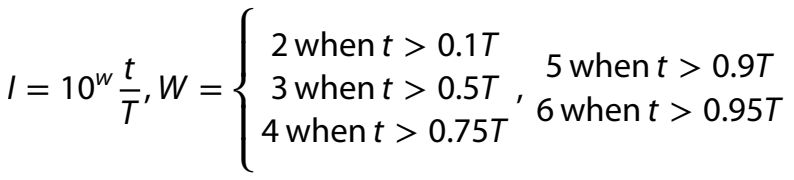

where $t$ is the current iteration, $T$ describes the maximum number of iteration, and $W$ stands for a fixed value.

The last step of hunting takes place when the ant is immersed in the sand. Then, the position of the antlion should be updated relative to the position where the ant hunted to increase the chances of a new hunt. The following equation describes this action:

Antlion $_{j}^{t}=\operatorname{Ant}_{i}^{t}$ iff $f\left(\operatorname{Ant}_{j}^{t}\right)<f\left(\right.$ Antlion $\left._{j}^{t}\right)$

Elitism is a feature of evolutionary algorithms that allows the best solution obtained to be maintained during the optimization process. In the ALO algorithm, the best antlion obtained at each step is stored and considered as an elite. The following equation shows the elite simulation:

$\mathrm{Ant}_{i}^{t}=\frac{R_{A}^{t}+R_{E}^{t}}{2}$

where $R_{A}^{t}$ is a random walk around the antlions by the roulette wheel in the $t$ th iteration, and $R_{E}^{t}$ describes also a random walk around the elite in the $t$ th iteration.

\subsection{MOALO algorithm}

Solving a multi-objective problem requires a set of solutions that create the best trade-off between the objectives, called the Pareto optimal set. The MOALO algorithm provides an archive for storing optimal solutions. By selecting a solution from the archive, the ALO algorithm can improve its quality. A leader is selected from the archive and used to improve the diversity of the archive. The archive must have limitations and the final solution to improve the distribution must be selected from the archive. Niching is used to measure the distribution of solutions in the archive. In this method, the neighborhood of each solution up to a predefined radius is checked to obtain the number of solutions that exist in the neighborhood of each solution. This is considered as a distribution criterion. Two mechanisms are used to improve the distribution of solutions in the archive. Firstly, the antlions are selected from the solutions that have a smaller population in their vicinity. The following equation defines the probability of selecting a solution from the archive:

$P_{i}=\frac{c}{N_{i}}$

where $c$ is the constant and must be greater than 1, and $N_{i}$ is the number of solutions in the neighborhood of the $i$ th solution.

Secondly, if the archive is full, the solutions that have a larger population in their vicinity should be removed from the archive and replaced by new solutions. The following equation indicates the probability of removing a solution from the archive:

$P_{i}=\frac{N_{i}}{c}$

where $c$ is the constant and must be greater than 1 , and $N_{i}$ is the number of solutions in the neighborhood of the $i$ th solution. Finally, the elite is selected from the archive using the roulette wheel and (19).

\subsection{Improved MOALO algorithm}

In this section, we propose an improved version of the MOALO algorithm (IMOALO) using opposition-based learning (OBL) and a self-adaptive population (SAP). 
Table 2 Parameters of metaheuristic algorithms

\begin{tabular}{|c|c|c|c|c|c|c|c|}
\hline \multicolumn{2}{|l|}{ MOPSO [24] } & \multicolumn{2}{|l|}{ NSGAII [25] } & \multicolumn{2}{|c|}{ MOALO \& IMOALO } & \multicolumn{2}{|c|}{ Common parameters } \\
\hline Parameters & Value & Parameters & Value & Parameters & Value & Parameters & Value \\
\hline nRep & 55 & pCrossover & 0.8 & ArchiveMaxSize & 100 & time slot & 24 \\
\hline W & 0.45 & nCrossover & 80 & Archive_X & 0 & VarMin & 0 \\
\hline Wdamp & 0.99 & pMutation & 0.5 & Archive_F & 1 & VarMax & 1 \\
\hline c1 & 0.5 & nMutation & 50 & $r$ & 0.7 & nPop & 100 \\
\hline c2 & 0.5 & $\mathrm{mu}$ & 0.02 & V_Max & 0.2 & Maxlt & 350 \\
\hline nGrid & 20 & sigma & 0.1 & - & - & - & - \\
\hline
\end{tabular}

Table 3 Statistical data for the total cost

\begin{tabular}{|c|c|c|c|c|c|}
\hline \multirow{2}{*}{\multicolumn{2}{|c|}{$\begin{array}{l}\text { Total cost } \\
\text { (cents) }\end{array}$}} & \multicolumn{4}{|c|}{ meta-heuristic algorithms } \\
\hline & & MOPSO [24] & NSGAII [25] & MOALO & IMOALO \\
\hline \multirow[t]{4}{*}{ RTP } & Min & 785.15 & 746.95 & 746.45 & 779.45 \\
\hline & Max & 2576.09 & 825.75 & 3760.95 & 3635.33 \\
\hline & Mean & 1012.68 & 779.57 & 1310.20 & 1183.57 \\
\hline & Var & $196,311.89$ & 518.95 & $1,004,040.18$ & $615,136.68$ \\
\hline \multirow[t]{4}{*}{ CPP } & Min & 1356 & 984.50 & 984.50 & 984.50 \\
\hline & Max & $11,665.66$ & 1929.50 & 2392.50 & 2224 \\
\hline & Mean & 3916.31 & 1402.93 & 1359.23 & 1407.02 \\
\hline & Var & $12,305,955.81$ & $81,232.72$ & $174,422.02$ & $105,689.56$ \\
\hline
\end{tabular}

OBL helps to produce a more diverse initial population, while the SPA automatically selects the population size at each iteration. Algorithm 1 shows the pseudocode of the IMOALO algorithm.

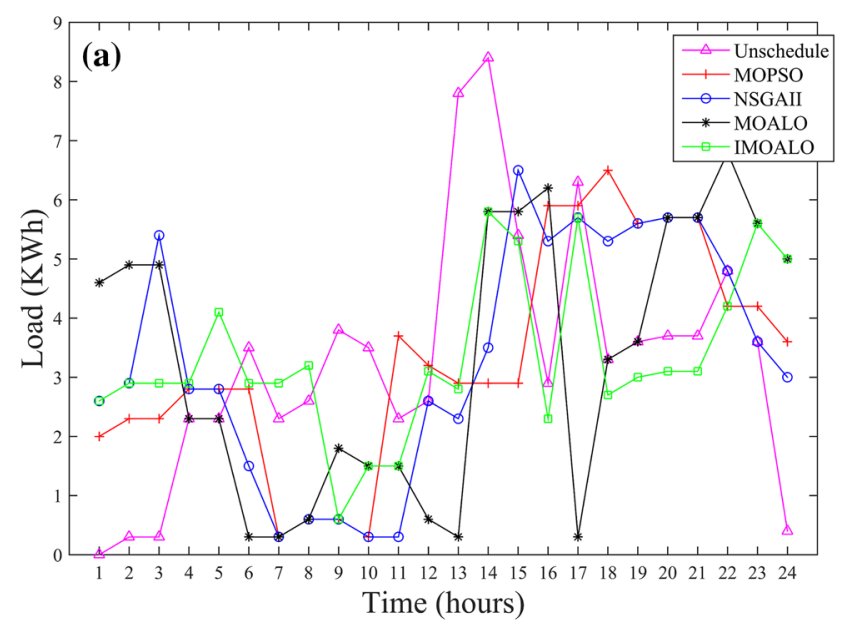

\subsubsection{Opposition based learning}

The MOALO algorithm, like other meta-heuristic optimization algorithms, requires a random initial population. If this initial population is close to the optimal solution, MOALO has more hope of moving towards the optimal solution. However, the initial solution may be far from the optimal solution. Also, the worst-case scenario is that the initial solution is in the opposite direction to the optimal solution. In this case, the optimization process may require more time or the optimal answer may not be achieved. Therefore, to increase the productivity of the initial population of the MOALO algorithm, we use OBL, which is modeled as follows:

$\bar{X}_{i}=X_{\max }+X_{\min }-X_{i}$

where $\bar{X}_{i}$ shows the opposite location of $X_{i}$, and $X_{\max }$ and $X_{\min }$ indicate the upper and lower bounds. If $\bar{X}_{i}$ has better fitness than $X_{i}$, then $\bar{X}_{i}$ replaces $X_{i}$.

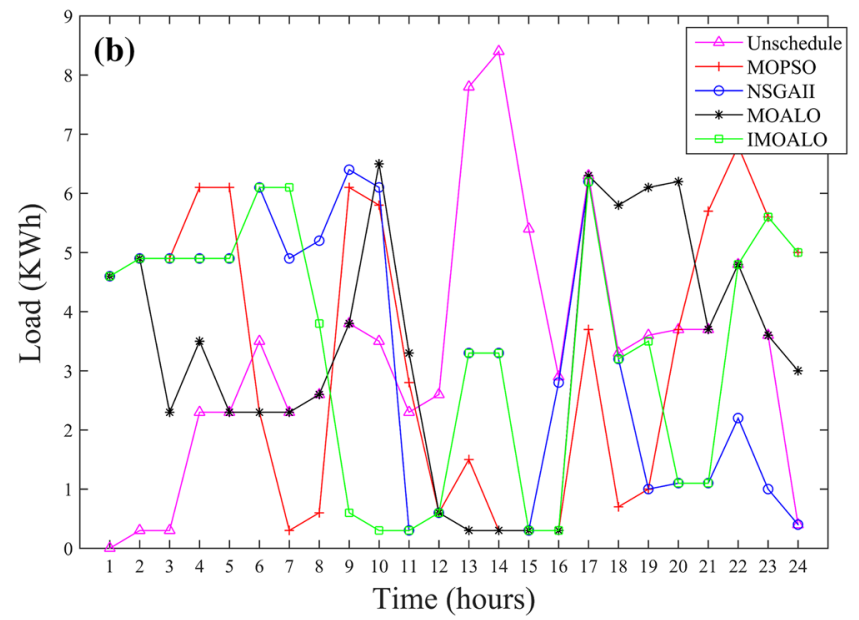

Fig. 3 Hourly load consumption a with RTP signal and b with CPP signal 

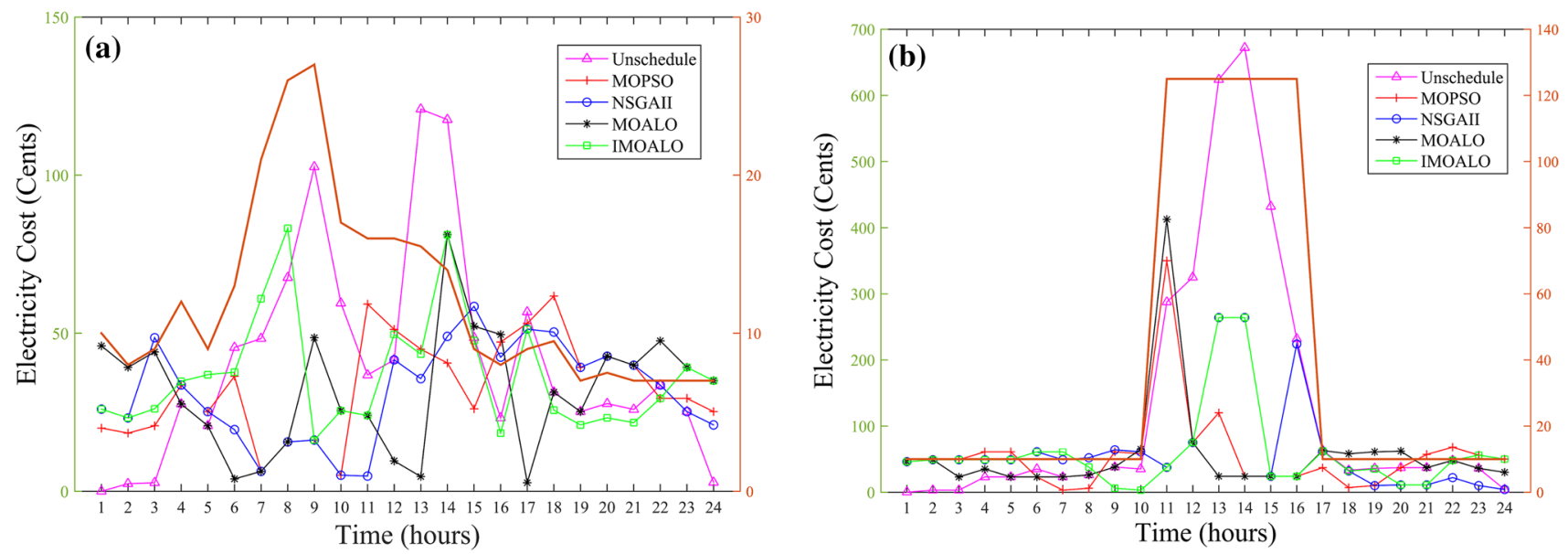

Fig. 4 Hourly electricity cost: $\mathbf{a}$ with the RTP signal and $\mathbf{b}$ with the CPP signal

\subsubsection{Self-adaptive population}

The MOGOA algorithm requires a control parameter to determine the population size. It should be noted that determining population size in specific optimization issues is a very difficult and challenging task. The self-organizing population does this automatically each time the algorithm is repeated. In the first, the initial population size is determined as follows:

pop $=10 * d$

where $d$ indicates the number of the variables. Then, in the next iteration of the main loop of the algorithm, the size of the new population is obtained as follows:

pop $_{\text {new }}=\operatorname{round}(\mathrm{pop}+r *$ pop $)$ where $r$ is a random number between $[-0.5,0.5]$. It is a control parameter that changes the size of the population and is produced randomly with a uniform distribution. Depending on whether the sign $r$ is negative or positive, the population size decreases or increases. When the new population size is larger than the size of the population

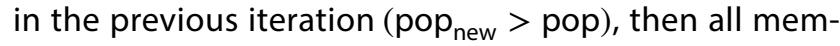
bers of the current population are transferred to the next iteration and the rest of the new population is produced according to elitism. But if the size of the new population is smaller than the population size of the previous iteration ( $_{\text {pop }}$ new $<$ pop), then only the top members of the current population are moved to the next iteration and the rest are removed. And when the new population size is less than the problem dimension, the size of the new population equals the number of variables in the problem.
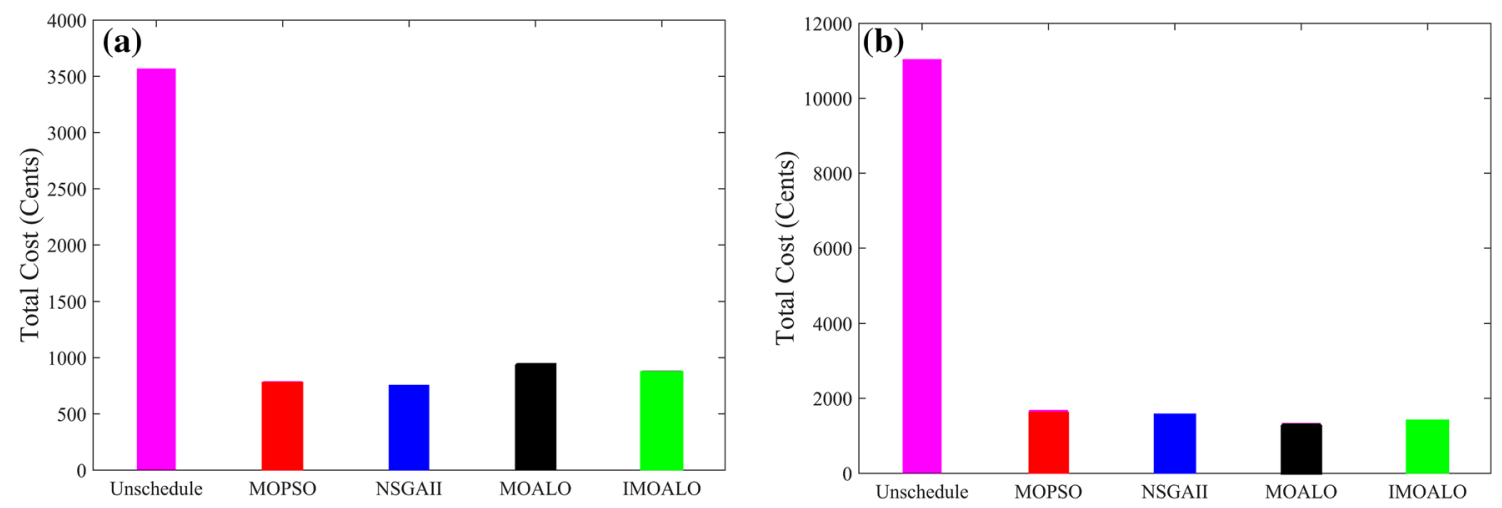

Fig. 5 Total cost per day a with an RTP signal b with a CPP signal 
Fig. 6 Histogram plot for the total cost a with RTP signal $\mathbf{b}$ with CPP signal

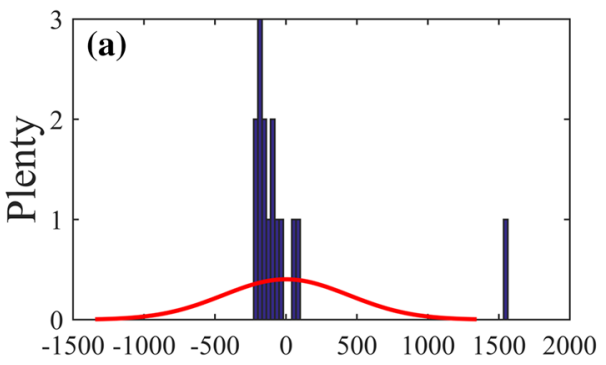

Error of total cost for MOPSO

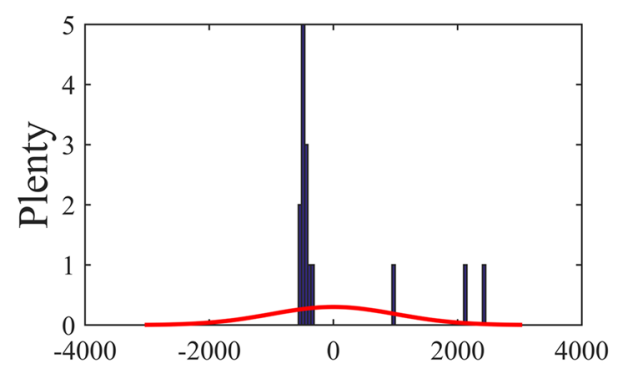

Error of total cost for MOALO
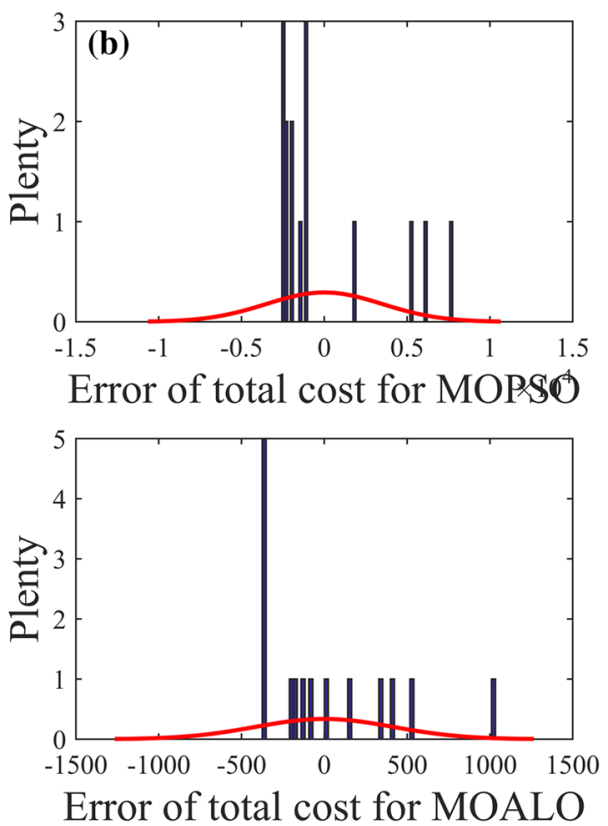
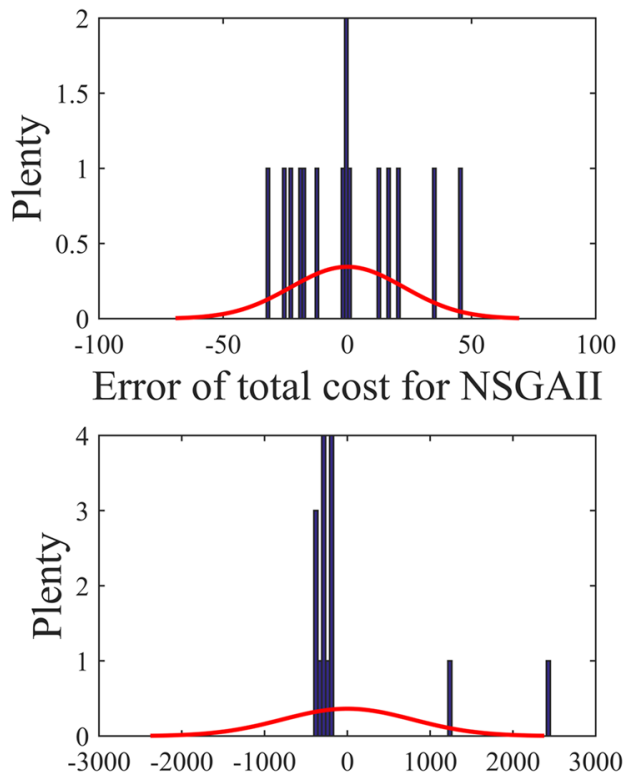

Error of total cost for IMOALO

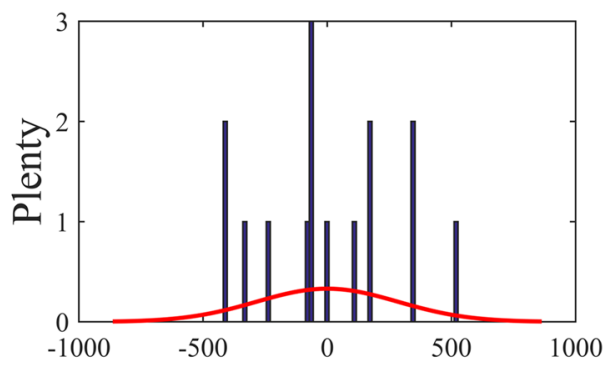

Error of total cost for NSGAII

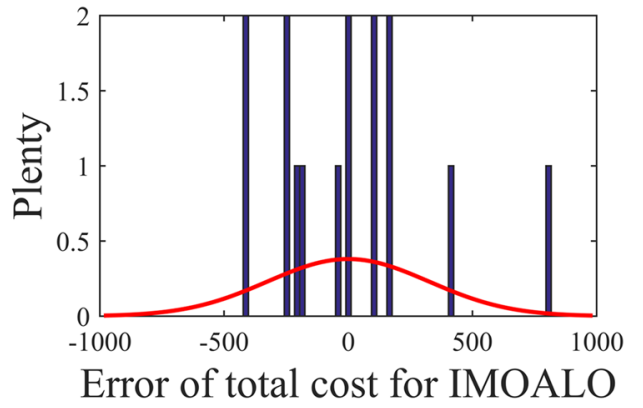



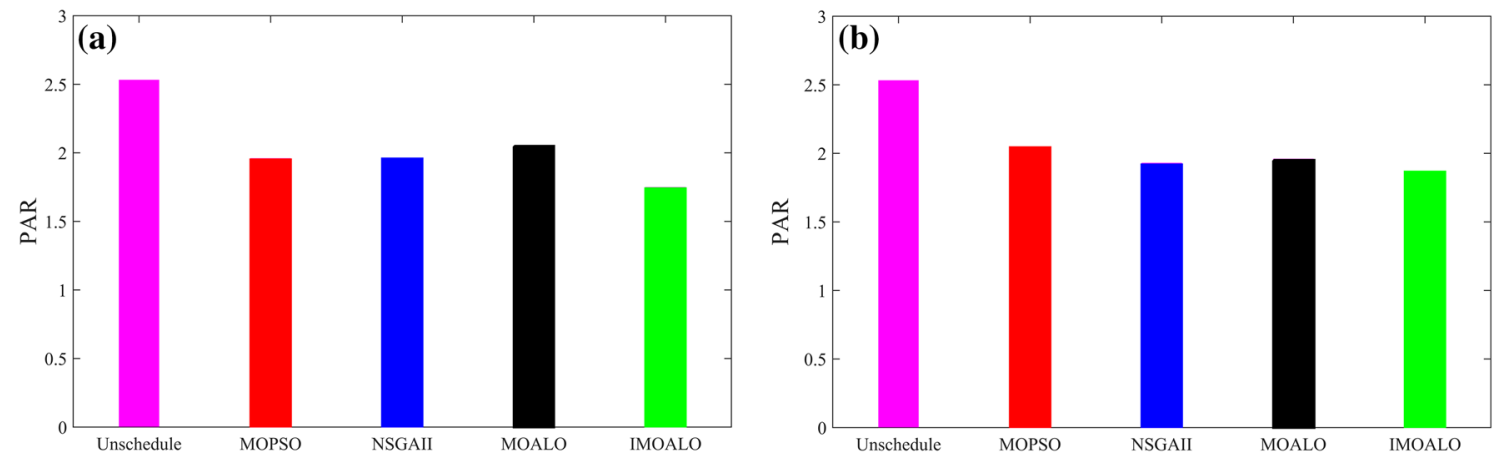

Fig. 7 PAR reduction a with an RTP signal b with a CPP signal

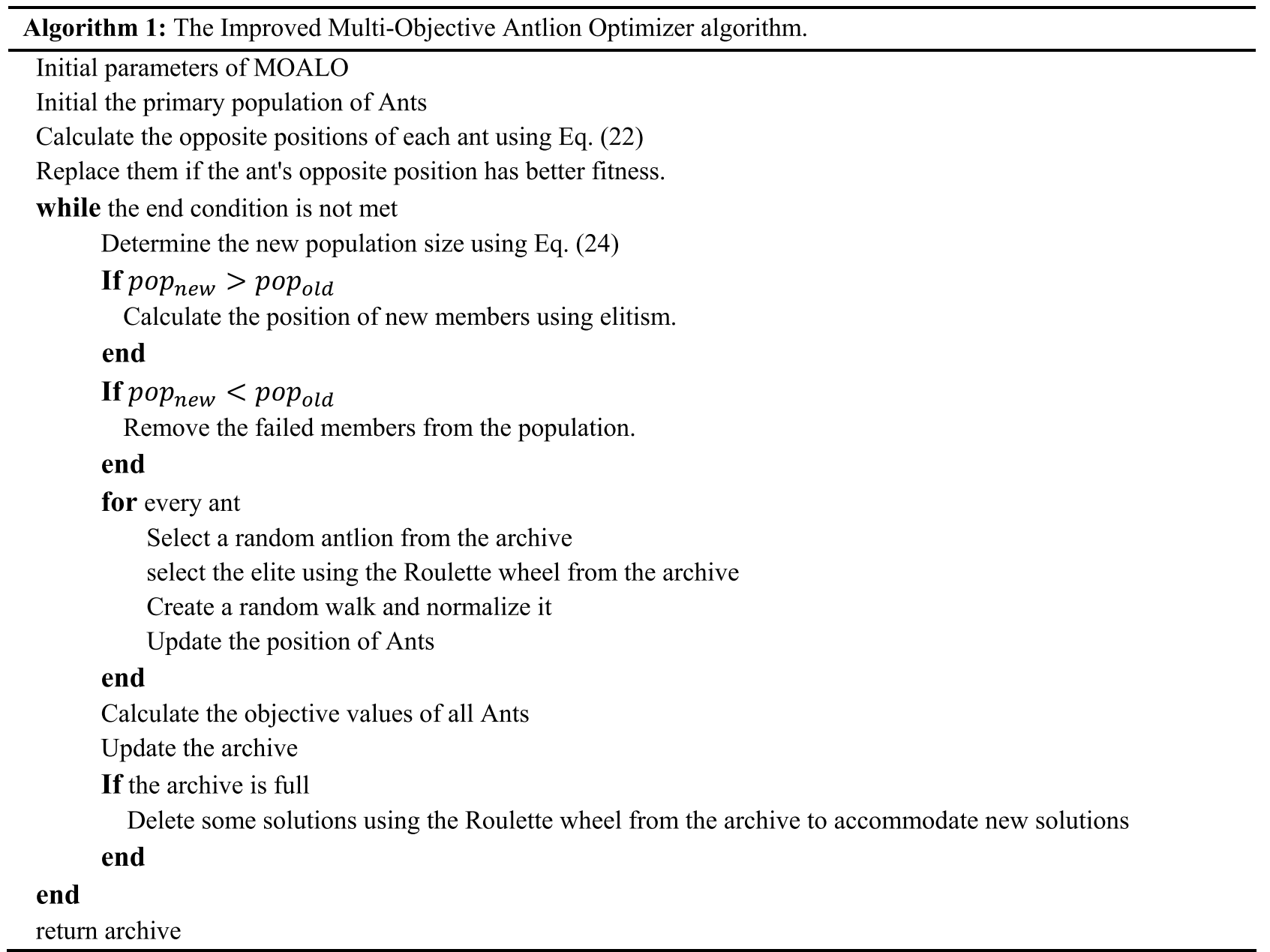


Fig. 8 Histogram plot for PAR a with an RTP signal $\mathbf{b}$ with CPP signal
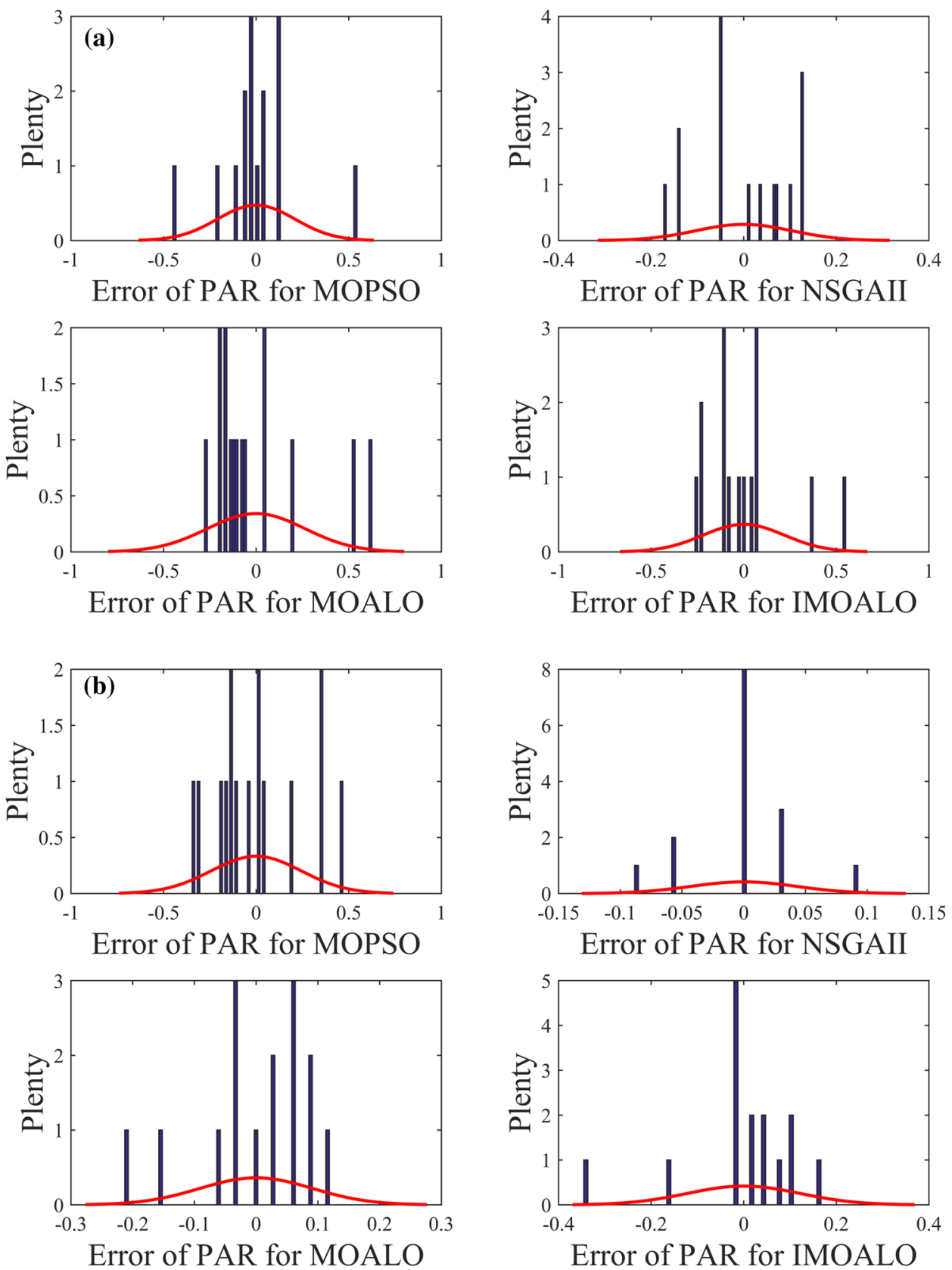

\section{Simulation and results}

In this section, we review the results of the optimization algorithms. As stated in the previous sections, the main purpose of this paper is to provide an optimal residential scheduling scheme to reduce electrical costs to increase consumer profitability and reduce PAR to increase power grid efficiency and stability; consumer waiting time is also reduced to increase user comfort. In the proposed scheme, a home with different appliances in terms of energy consumption and operational time is considered. RTP and CPP signals are used as the electricity tariff, which is a good solution for increasing the reliability and stability of the power grid. The proposed MOALO meta-heuristic algorithm was simulated in MATLAB software Ver.2015a using a processor installed with Intel ${ }^{\circledR}$ Core $^{\mathrm{TM}}$ i7-4500U CPU @ $1.8 \mathrm{GHz} 2.4 \mathrm{GHz}$ and installed memory Ram $8 \mathrm{~GB}$ on Windows platform. The other three algorithms MOPSO [24], NSGAII [25], and MOALO have also been simulated to confirm the accuracy of the results of the proposed algorithm by comparing their results. Table 2 presents the parameters of meta-heuristic algorithms. To ensure fairness between the proposed IMOALO algorithm and other meta-heuristic algorithms, each algorithm is run fifty times 
Table 4 Statistical data for PAR

\begin{tabular}{llllll}
\hline PAR & \multicolumn{4}{l}{ Meta-heuristic algorithms } \\
\cline { 3 - 6 } & & MOPSO [24] & NSGAII [25] & MOALO & IMOALO \\
\hline RTP & Min & 1.47 & 1.68 & 1.71 & 1.71 \\
& Max & 2.46 & 1.98 & 2.61 & 2.52 \\
& Mean & 1.92 & 1.85 & 1.99 & 1.97 \\
& Var & 0.04 & 0.01 & 0.06 & 0.04 \\
CPP & Min & 1.68 & 1.83 & 1.68 & 1.53 \\
& Max & 2.49 & 2.01 & 2.01 & 2.04 \\
& Mean & 2.03 & 1.92 & 1.89 & 1.88 \\
& Var & 0.05 & 0.0018 & 0.08 & 0.01 \\
\hline
\end{tabular}

with the same initial population. Table 3 shows the data obtained from this simulation, which includes the total cost and PAR for each meta-heuristic algorithm.

We assumed a home in which electrical appliances are divided into three categories: shiftable, non-shiftable, and fixed appliances. The proposed scheme for shiftable appliances has been implemented. A comparative analysis is performed for the schedule obtained from the proposed algorithm and other meta-heuristic algorithms, and the following results show that the proposed algorithm has a good performance to achieve the above objectives. Figure (2) shows the RTP and CPP signals used in this scheme.

Under these tariffs, the cost of electricity consumption during peak hours increases sharply. The purpose of this increase is to encourage consumers to reduce consumption during peak hours. As shown in Fig. (2), the cost of electricity consumption during off-peak hours is less than 20 cents for CPP tariff, while at 11 to $16 \mathrm{~h}$, which is peak hours, the cost of electricity has increased by more than 120 cents. Therefore, the consumer has to pay a lot of money during peak hours. Also, the power grid equipment suffers a lot of depreciation. The hourly load consumption of scheduled and unscheduled patterns for CPP and RTP tariffs is shown in Fig. (3).

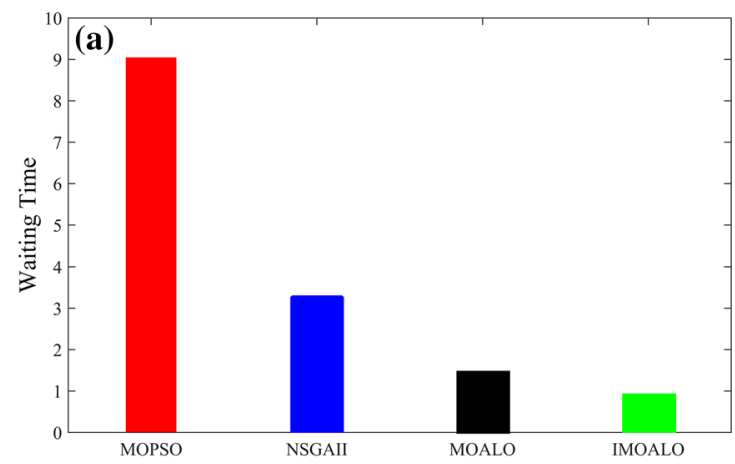

Fig. 9 Waiting time reduction a with an RTP signal. b With CPP signal
According to Fig. (3), the unscheduled pattern has a peak in the period of 13 and 14 , which is equal to 7.5 and $8.5 \mathrm{KW}$, respectively, and are formed during peak hours. the proposed IMOALO algorithm has a good performance in terms of shifting loads to off-peak hours using both RTP and CPP signals. For this algorithm, the total load consumed during peak hours is about $14 \mathrm{~kW}$ and $11 \mathrm{KW}$ using RTP and CPP, respectively, while the load demand during peak hours for the unscheduled pattern is $25 \mathrm{KW}$ and $28 \mathrm{~kW}$ using RTP and CPP, respectively. Thus, IMOALO has been able to reduce demand during peak hours by more than $45 \%$ and $60 \%$ for RTP and CPP signals, respectively, compared to the unscheduled pattern. Also, this algorithm has been able to distribute the load demanded by the consumer in a balanced way outside the peak hours, and fortunately, we do not see a peak in the time slots during the peak hours for the pattern optimized by IMOALO. Also, the peak of scheduled patterns is less compared to unscheduled patterns. Other meta-heuristic algorithms also perform better than unscheduled. Figure (4) illustrates the hourly electricity cost along with a plot of the RTP and CPP signals.

The results in Fig. (4) shows that each algorithm tries to schedule the cost in off-peak hours and the electricity cost of the patterns scheduled by the MOALO, NSGAIl, MOPSO, and IMOALO algorithms are much less than the unscheduled pattern. Also, according to Fig. (4b) the proposed IMOALO algorithm costs much less than the other three algorithms. The unscheduling during 13th and 14th give a peak of more than 100 and 600 cents for RTP and CPP signals, respectively. This peak has been reduced by up to $50 \%$ by MOPSO, NSGAll, and MOALO algorithms using the CPP signal, while the proposed IMOALO algorithm has reduced this peak close to zero. The total cost of electricity per day using RTP and CPP signals for the patterns scheduled by the optimization algorithms and the unscheduled pattern is estimated in Fig. (5).

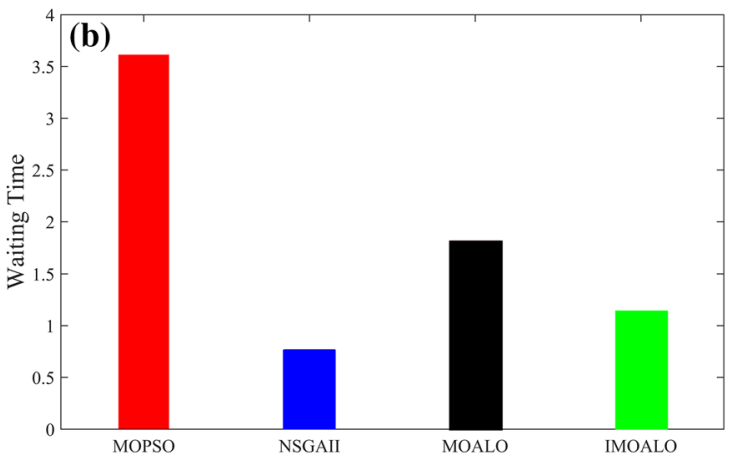


Fig. 10 Histogram plot for waiting time a with RTP signal b with CPP signal
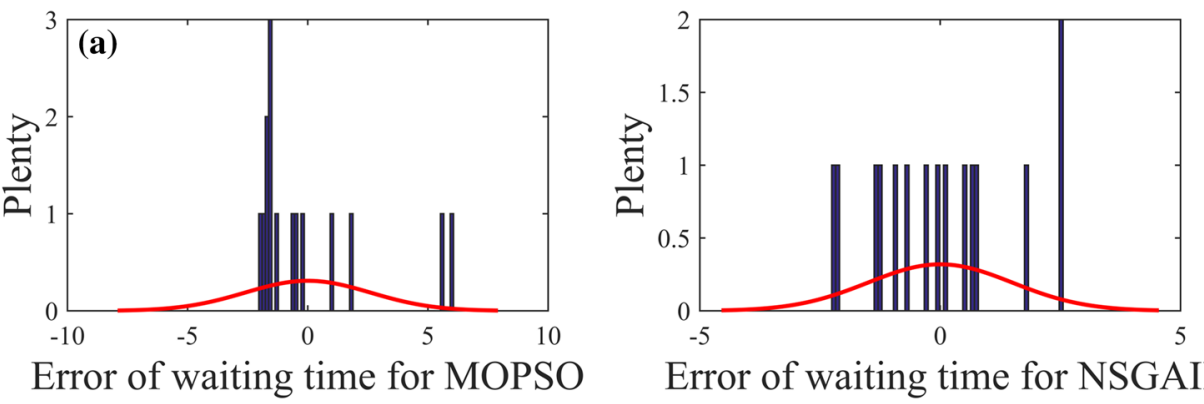

Error of waiting time for NSGAII

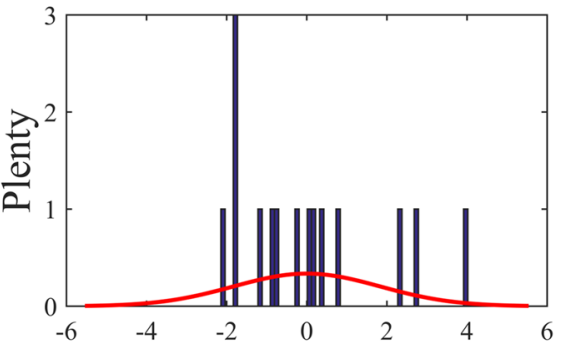

Error of waiting time for MOALO

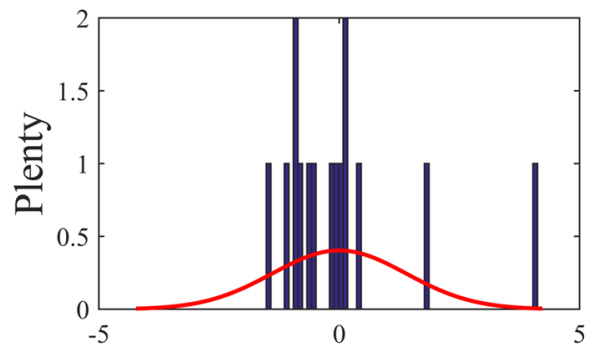

Error of waiting time for IMOALO

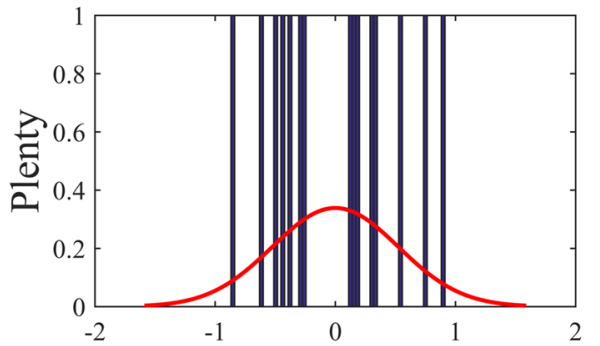

Error of waiting time for NSGAII

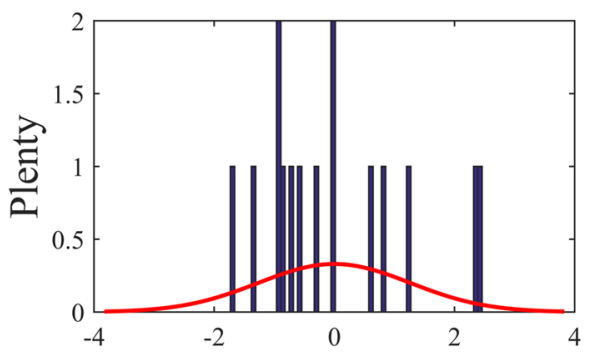

Error of waiting time for IMOALO
According to the results in Fig. (5), the total electrical cost for the unscheduled pattern is 3500 and 11,000 cents using RTP and CPP signals, respectively. All the meta-heuristic algorithms used in this simulation have succeeded in reducing these costs and have performed similarly in this regard. All scheduled patterns have reduced the total cost by up to $75 \%$ and $80 \%$, respectively, using RTP and CPP signals. These results show that the difference between the unscheduled pattern and other patterns is too much. According to Figs. 3 and 4, this difference is due to the fact that the energy consumed during peak hours by the unscheduled pattern is much higher than other patterns.
For example, when using the CPP tariff, the load consumed by the unscheduled pattern during peak hours is about $28 \mathrm{kWh}$, while for other patterns, it is less than $10 \mathrm{~kW}$. Besides, the proposed IMOALO algorithm has had the best performance when it uses the CPP signal as the electricity tariff. The proposed algorithm performed better than the MOALO algorithm when using RTP as the cost of electricity. Figure (6) shows a histogram of the total cost data in Table 3. Also, the minimum, maximum, mean, and variance values for the total cost are given in Table 3.

Figure (6) and Table 3 show that when reducing the total cost, the variance for the IMOALO algorithm is $615,136.68$ 
Table 5 Statistical data for waiting time

\begin{tabular}{llllll}
\hline PAR & \multicolumn{4}{l}{ Meta-heuristic algorithms } \\
\cline { 3 - 6 } & & MOPSO [24] & NSGAII [25] & MOALO & IMOALO \\
\hline RTP & Min & 0.93 & 0.29 & 0.07 & 0.29 \\
& Max & 9.03 & 5.10 & 6.23 & 5.93 \\
& Mean & 2.97 & 2.54 & 2.21 & 1.80 \\
& Var & 6.80 & 2.25 & 3.34 & 1.94 \\
CPP & Min & 0.81 & 0.25 & 0.35 & 0.45 \\
& Max & 7.35 & 2.03 & 5.87 & 4.64 \\
& Mean & 2.82 & 1.11 & 2.03 & 2.18 \\
& Var & 3.66 & 0.27 & 1.71 & 1.59 \\
\hline
\end{tabular}

and 105,689.56 using RTP and CPP, respectively, which is less than the variance obtained for the basic IMOALO algorithm. This indicates that IMOALO has a more robust performance than MOALO. Also, this value is 518.95 and $81,232.72$ for the NSGAll algorithm, respectively, using RTP and CPP signals. Therefore, the NSGAll algorithm is much more robust than the other three algorithms. But, the minimum total cost for the IMOALO algorithm is 984.50 cents, which is less than the MOPSO algorithm and is equal to NSGAll. Also, the average value for the IMOALO algorithm has the same salvation as the other three algorithms. Therefore, the proposed algorithm performs better than other algorithms in reducing total cost. Figure (7) shows the performance of all scheduled and unscheduled models in terms of PAR reduction According to RTP and CPP signals.

According to Fig. (7), the PAR value for the unscheduled pattern is 2.5. All programmed patterns have been able to reduce this value well for both RTP and CPP cost signals. In the meantime, our proposed pattern has performed better than others. IMOALO not only reduces the PAR obtained in the MOALO scheme, but also the PAR obtained using this scheme is less than other schemes. IMOALO was able to reduce the PAR value by $35 \%$ and $30 \%$, respectively, using RTP and CPP signals. A histogram of the PAR is shown in Fig. (8). Also, Table 4 presents the minimum, maximum, mean, and variance values for the PAR.

Table 4 shows that the variance for our proposed IMOALO algorithm is 0.04 and 0.01 using RTP and CPP signals, respectively, which is less than the variance obtained for MOALO and shows that the proposed algorithm is more robust than MOALO in terms of PAR reduction. The minimum PAR for the IMOALO algorithm is 1.53 using CPP, which is less than the value obtained for other algorithms. But when using the RTP signal, MOPSO got the lowest value, which is 1.47 . Figure (9) presents the waiting time for each algorithm using RTP and CPP signal.

According to Fig. (9), the MOPSO algorithm has the longest waiting time for both RTP and CPP signals, which are 9 and 3.6, respectively. Our proposed IMOALO algorithm has the best performance in terms of reducing the waiting time when using RTP as an electricity tariff. The waiting time for IMOALO is 1 , which is 8 units less than the waiting time obtained by the MOPSO algorithm using the RTP signal. When using the CPP signal, NSGAll performs better than others. The waiting time for this pattern is less than one unit. Then, our proposed algorithm has a waiting time of 1.3 units. Figure (10) shows a histogram of the waiting time data in Table 3. Also, the minimum, maximum, mean, and variance values for the waiting time are given in Table 5.

According to Fig. (10) and the data in Table 5, when using the RTP tariff, the variance and the mean for our proposed plan are 1.94 and 1.8 , respectively, which is less than the variance and the mean obtained for other plans and that means IMOALO performed better. But when using the

Fig. 11 The running time

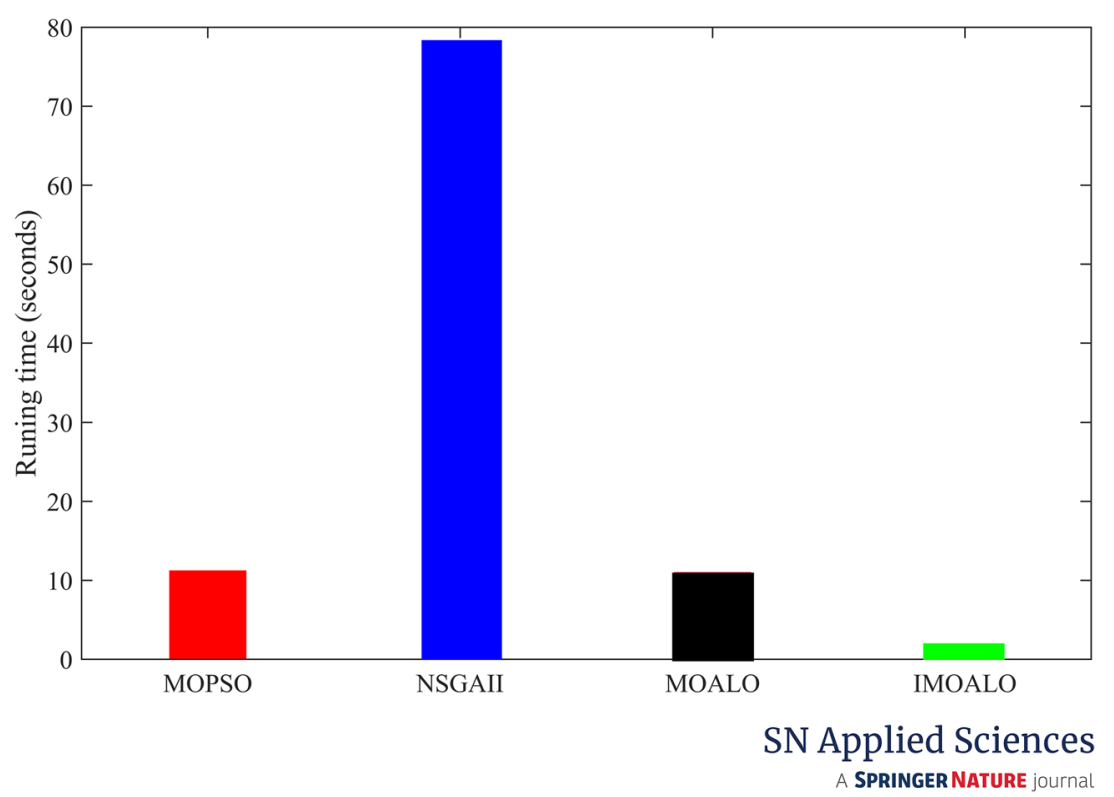


Fig. 12 Pareto optimal front obtained by the IMOALO algorithm. a With an RTP signal. b With CPP signal
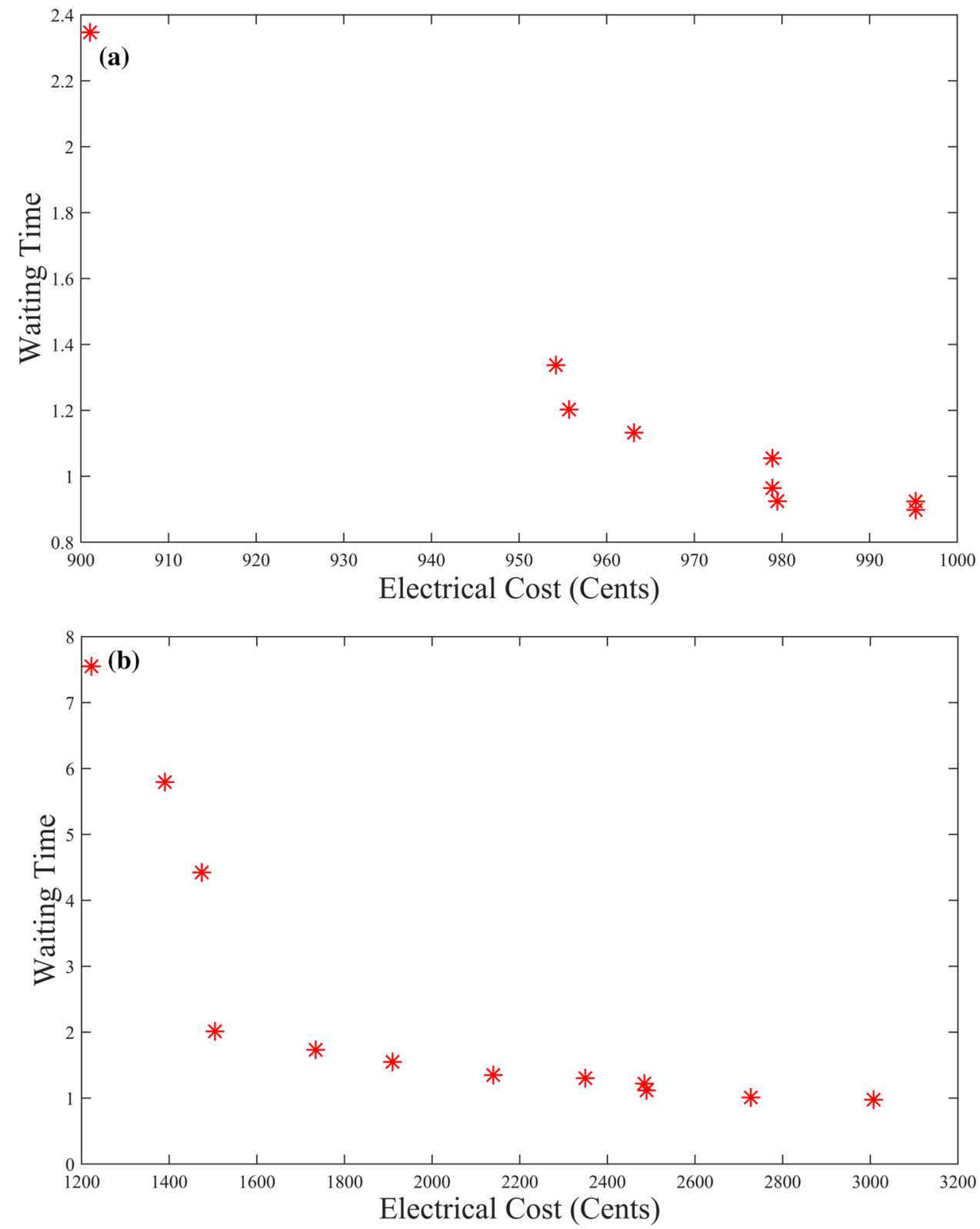

CPP tariff, NSGAll has the lowest variance and the mean, which is 0.27 and 1.11 , respectively. Figure (11) presents the running time for each algorithm.

According to Fig. (11), the NSGAll algorithm has the longest running time, which is more than $70 \mathrm{~s}$, but this is not good. The running time for the proposed IMOALO algorithm and MOPSO and MOALO algorithms is less than $20 \mathrm{~s}$, which seems appropriate. The IMOALO algorithm has the shortest running time. Figure (12) shows the Pareto optimal front for the proposed IMOALO algorithm.

According to Fig. (12), the repository obtained for the proposed IMOALO algorithm had 9 and 12 members, respectively, when using the RTP and CPP signals.
Based on the results presented in this section, the proposed IMOALO algorithm has performed well in designing an optimal residential schedule. This scheme can significantly reduce the total cost and PAR and increases the user comfort; therefore, the implementation of this plan will greatly reduce the electricity bill of consumers and also increase the stability of the power grid and reduce the maintenance costs that the company has to pay. 


\section{Conclusion}

Optimal consumption and management of electricity help to reduce consumer demand. Demand-side management not only reduces electricity costs and increases consumer profitability, but also reduces network maintenance costs. In this paper, the IMOALO meta-heuristic algorithm is presented to optimize the consumption pattern of home appliances. The proposed scheme classifies the devices into three categories: shiftable, non-shiftable, and fixed. RTP and CPP signal is considered as electricity consumption tariff, which is a good solution to increase grid efficiency and stability. The simulation results are compared with MOPSO, MOALO, and NSGAll algorithms. The IMOALO algorithm has been able to reduce the daily cost of electricity by up to $75 \%$ and $80 \%$, respectively, using RTP and CPP signals, and PAR by up to $35 \%$ and $30 \%$, respectively, using RTP and CPP signals compared to the unscheduled pattern, which has performed better than other meta-heuristic algorithms. This algorithm also reduces the waiting time compared to other algorithms. Therefore, the results show that the use of the proposed plan can be an effective solution to deal with the growing consumer demand.

\section{Compliance with ethical standards}

Conflict of interest The authors declare no conflict of interest.

\section{References}

1. Ullah I, Kim D (2017) An improved optimization function for maximizing user comfort with minimum energy consumption in smart homes. Energies 10(11):1818

2. Alizadeh E, Barzegari M, Momenifar M, Ghadimi M, Saadat S (2016) Investigation of contact pressure distribution over the active area of PEM fuel cell stack. Int J Hydrog Energy 41(4):3062-3071

3. Wang B, Zhao D, Li W, Wang Z, Huang Y, You Y, Becker S (2020) Current technologies and challenges of applying fuel cell hybrid propulsion systems in unmanned aerial vehicles. Prog Aerosp Sci 116:100620

4. Meyabadi AF, Deihimi MH (2017) A review of demand-side management: reconsidering theoretical framework. Renew Sustain Energy Rev 80:367-379

5. Yi W, Dong W (2015) Modeling and simulation of discharging characteristics of external melt ice-on coil storage system. Int J Smart Home 9(2):179-192

6. Yu D et al (2019) System identification of PEM fuel cells using an improved Elman neural network and a new hybrid optimization algorithm. Energy Rep 5:1365-1374

7. CaoY et al (2019) Experimental modeling of PEM fuel cells using a new improved seagull optimization algorithm. Energy Rep 5:1616-1625

8. Mariano-Hernández D, Hernández-Callejo L, Zorita-Lamadrid A, Duque-Pérez O, García FS (2020) A review of strategies for building energy management system: model predictive control, demand side management, optimization, and fault detect \& diagnosis. J Build Eng 33:101692
9. Zehir MA, Bagriyanik M (2012) Demand side management by controlling refrigerators and its effects on consumers. Energy Convers Manag 64:238-244

10. Chauhan RK, Chauhan K (2020) Impact of demand-side management system in autonomous DC microgrid. In: Abdel Aleem SHE, Abdelaziz AY, Zobaa AF, Bansal R (eds) Decision making applications in modern power systems. Elsevier, Amsterdam, pp 389-410

11. Liu Q, Dannah W, Liu X (2019) "Intelligent algorithms in home energy management systems: a survey". In 2019 IEEE SmartWorld, Ubiquitous Intelligence \& Computing, Advanced \& Trusted Computing, Scalable Computing \& Communications, Cloud \& Big Data Computing, Internet of People and Smart City Innovation (SmartWorld/SCALCOM/UIC/ATC/CBDCom/IOP/SCI), pp. 296-299. IEEE

12. Bharathi C, Rekha D, Vijayakumar V (2017) Genetic algorithm based demand side management for smart grid. WirelPersCommun 93(2):481-502

13. Essiet IO, Sun Y, Wang Z (2019) Optimized energy consumption model for smart home using improved differential evolution algorithm. Energy 172:354-365

14. Shakouri H, Kazemi A (2017) Multi-objective cost-load optimization for demand side management of a residential area in smart grids. Sustain Cities Soc 32:171-180

15. Hussain HM, Javaid N, Iqbal S, Hasan QU, Aurangzeb K, Alhussein M (2018) An efficient demand side management system with a new optimized home energy management controller in smart grid. Energies 11(1):190

16. Marzband M, Ghazimirsaeid SS, Uppal H, Fernando T (2017) A real-time evaluation of energy management systems for smart hybrid home Microgrids. Electr Power Sys Res 143:624-633

17. Bera S, Misra S, Chatterjee D (2017) C2C: community-based cooperative energy consumption in smart grid. IEEE Trans Smart Grid 9(5):4262-4269

18. Cao Y, et al. (2019) "Multi-objective optimization of a PEMFC based CCHP system by meta-heuristics". Energy Reports

19. Lokeshgupta B, Sivasubramani S (2019) Cooperative game theory approach for multi-objective home energy management with renewable energy integration. IET Smart Grid 2(1):34-41

20. Vardakas JS, Zorba N, Verikoukis CV (2014) A survey on demand response programs in smart grids: pricing methods and optimization algorithms. IEEE CommunSurv Tutor 17(1):152-178

21. Shuja SM et al (2019) Efficient scheduling of smart home appliances for energy management by cost and PAR optimization algorithm in smart grid. In: Barolli L, Takizawa $M$, Xhafa $F$, Enokido T (eds) Workshops of the international conference on advanced information networking and applications. Springer, New York, pp 398-411

22. Lee JY, Choi SG (2014) "Linear programming based hourly peak load shaving method at home area". In 16th international conference on advanced communication technology, pp. 310-313. IEEE

23. Fei $H$, Li Q, Sun D (2017) A survey of recent research on optimization models and algorithms for operations management from the process view. Sci Program 2017:1-19

24. Hossain MA, Pota HR, Squartini S, Abdou AF (2019) Modified PSO algorithm for real-time energy management in grid-connected microgrids. Renew Energy 136:746-757

25. Sofia AS, GaneshKumar P (2018) Multi-objective task scheduling to minimize energy consumption and makespan of cloud computing using NSGA-II. J NetwSystManag 26(2):463-485

Publisher's Note Springer Nature remains neutral with regard to jurisdictional claims in published maps and institutional affiliations. 